\title{
The modified general iterative methods for nonexpansive semigroups in banach spaces ${ }^{\dagger}$
}

\author{
Rabian Wangkeeree ${ }^{*}$ and Pakkapon Preechasilp
}

* Correspondence: rabianw@nu.ac. th

Department of Mathematics, Faculty of Science, Naresuan University, Phitsanulok 65000, Thailand

\section{Abstract}

In this paper, we introduce the modified general iterative approximation methods for finding a common fixed point of nonexpansive semigroups which is a unique solution of some variational inequalities. The strong convergence theorems are established in the framework of a reflexive Banach space which admits a weakly continuous duality mapping. The main result extends various results existing in the current literature.

Mathematics Subject Classification (2000) 47H05, 47H09, 47J25, 65J15

Keywords: nonexpansive semigroups, strong convergence theorem, Banach space, common fixed point

\section{Introduction}

Let $C$ be a nonempty subset of a normed linear space $E$. Recall that a mapping $T$ : $C \rightarrow$ $C$ is called nonexpansive if

$$
\|T x-T y\| \leq\|x-y\|, \quad \forall x, y \in E .
$$

We use $F(T)$ to denote the set of fixed points of $T$, that is, $F(T)=\{x \in E: T x=x\}$. A self mapping $f: E \rightarrow E$ is a contraction on $E$ if there exists a constant $\alpha \in(0,1)$ and $x$, $y \in E$ such that

$$
\|f(x)-f(y)\| \leq \alpha\|x-\gamma\| .
$$

We use $\Pi_{E}$ to denote the collection of all contractions on $E$. That is, $\Pi_{E}=\{f: f$ is a contraction on $E$ \}.

Here, we consider a scheme for a semigroup of nonexpansive mappings. Let $C$ be a closed convex subset of a Banach space $E$. Then, a family $\mathcal{S}=\{T(s): 0 \leq s<\infty\}$ of mappings of $C$ into itself is called a nonexpansive semigroup on $E$ if it satisfies the following conditions:

(i) $T(0) x=x$ for all $x \in C$;

(ii) $T(s+t)=T(s) T(t)$ for all $s, t \geq 0$;

(iii) $\|T(s) x-T(s) y\| \leq\|x-y\|$ for all $x, y \in C$ and $s \geq 0$;

(iv) for all $x \in C$, the mapping $s \mapsto T(s) x$ is continuous.

We denote by $F(\mathcal{S})$ the set of all common fixed points of $\mathcal{S}$, that is,

\section{Springer}

(C) 2011 Wangkeeree and Preechasilp; licensee Springer. This is an Open Access article distributed under the terms of the Creative Commons Attribution License (http://creativecommons.org/licenses/by/2.0), which permits unrestricted use, distribution, and reproduction in any medium, provided the original work is properly cited. 


$$
F(\mathcal{S}):=\{x \in E: T(s) x=x, 0 \leq s<\infty\}=\cap_{s \geq 0} F(T(s)) .
$$

One classical way to study nonexpansive mappings is to use contractions to approximate a non-expansive mapping ([1-3]). More precisely, take $t \in(0,1)$ and define a contraction $T_{t}: E \rightarrow E$ by

$$
T_{t} x=t u+(1-t) T x, \quad \forall x \in E,
$$

where $u \in E$ is a fixed point. Banach's contraction mapping principle guarantees that $T_{t}$ has a unique fixed point $x_{t}$ in $E$. It is unclear, in general, what is the behavior of $x_{t}$ as $t \rightarrow 0$, even if $T$ has a fixed point. However, in the case of $T$ having a fixed point, Browder [1] proved that if $E$ is a Hilbert space, then $x_{t}$ converges strongly to a fixed point of $T$. Reich [2] extended Browder's result to the setting of Banach spaces and proved that if $E$ is a uniformly smooth Banach space, then $\left\{x_{t}\right\}$ converges strongly to a fixed point of $T$ and the limit defines the (unique) sunny nonexpansive retraction from $E$ onto $F(T) . \mathrm{Xu}$ [3] proved Reich's results hold in reflexive Banach spaces which have a weakly continuous duality mapping.

In the last ten years or so, the iterative methods for nonexpansive mappings have recently been applied to solve convex minimization problems; see, e.g., [4-6] and the references therein.

By a gauge function $\phi$, we mean a continuous strictly increasing function $\phi:[0, \infty) \rightarrow$ $[0, \infty)$ such that $\phi(0)=0$ and $\phi(t) \rightarrow \infty$ as $t \rightarrow \infty$. Let $E^{*}$ be the dual space of $E$. The duality mapping $J_{\varphi}: E \rightarrow 2^{E^{*}}$ associated with a gauge function $\phi$ is defined by

$$
J_{\varphi}(x)=\left\{f^{*} \in E^{*}:\left\langle x, f^{*}\right\rangle=\|x\| \varphi(\|x\|), \quad\left\|f^{*}\right\|=\varphi(\|x\|)\right\}, \quad \forall x \in E .
$$

In particular, the duality mapping with the gauge function $\phi(t)=t$, denoted by $J$, is referred to as the normalized duality mapping. Clearly, there holds the relation $J_{\varphi}(x)=\frac{\varphi(\|x\|)}{\|x\|} J(x)$ for all $x \neq 0$ (see [7]).

Browder [7] initiated the study of certain classes of nonlinear operators by means of the duality mapping $J_{\phi}$. Following Browder [7], we say that a Banach space $E$ has a weakly continuous duality mapping if there exists a gauge $\phi$ for which the duality mapping $J_{\phi}(x)$ is single-valued and continuous from the weak topology to the weak" topology, that is, for any $\left\{x_{n}\right\}$ with $x_{n} \rightarrow x$, the sequence $\left\{J_{\phi}\left(x_{n}\right)\right\}$ converges weakly* to $J_{\phi}(x)$. It is known that $l^{p}$ has a weakly continuous duality mapping with a gauge function $\phi(t)$ $=t^{p-1}$ for all $1<p<\infty$. Set

$$
\Phi(t)=\int_{0}^{t} \varphi(\tau) \mathrm{d} \tau, \quad \forall t \geq 0,
$$

then

$$
J_{\varphi}(x)=\partial \Phi(\|x\|), \quad \forall x \in E,
$$

where $\partial$ denotes the sub-differential in the sense of convex analysis.

In a Banach space $E$ having a weakly continuous duality mapping $J_{\phi}$ with a gauge function $\phi$, an operator $A$ is said to be strongly positive [8] if there exists a constant $\bar{\gamma}>0$ with the property 


$$
\left\langle A x, J_{\varphi}(x)\right\rangle \geq \bar{\gamma}\|x\| \varphi(\|x\|)
$$

and

$$
\|\alpha I-\beta A\|=\sup _{\|x\| \leq 1}\left|\left\langle(\alpha I-\beta A) x, J_{\varphi}(x)\right\rangle\right|, \quad \alpha \in[0,1], \beta \in[-1,1],
$$

where $I$ is the identity mapping. If $E:=H$ is a real Hilbert space, then the inequality (1.4) reduces to

$$
\langle A x, x\rangle \geq \bar{\gamma}\|x\|^{2} \text { for all } x \in H .
$$

A typical problem is to minimize a quadratic function over the set of the fixed points of a nonexpansive mapping on a real Hilbert space $H$ :

$$
\min _{x \in C} \frac{1}{2}\langle A x, x\rangle-\langle x, b\rangle
$$

where $C$ is the fixed point set of a nonexpansive mapping $T$ on $H$ and $b$ is a given point in $H$. In 2003, Xu ([5]) proved that the sequence $\left\{x_{n}\right\}$ defined by the iterative method below, with the initial guess $x_{0} \in H$ chosen arbitrarily:

$$
x_{n+1}=\left(I-\alpha_{n} A\right) T x_{n}+\alpha_{n} u, \quad n \geq 0,
$$

converges strongly to the unique solution of the minimization problem (1.7) provided the sequence $\left\{\alpha_{n}\right\}$ satisfies certain conditions. Using the viscosity approximation method, Moudafi [9] introduced the following iterative iterative process for nonexpansive mappings (see $[10,11]$ for further developments in both Hilbert and Banach spaces). Let $f$ be a contraction on $H$. Starting with an arbitrary initial $x_{0} \in H$, define a sequence $\left\{x_{n}\right\}$ recursively by

$$
x_{n+1}=\left(1-\sigma_{n}\right) T x_{n}+\sigma_{n} f\left(x_{n}\right), \quad n \geq 0,
$$

where $\left\{\sigma_{n}\right\}$ is a sequence in $(0,1)$. It is proved $[9,11]$ that under certain appropriate conditions imposed on $\left\{\sigma_{n}\right\}$, the sequence $\left\{x_{n}\right\}$ generated by (1.9) strongly converges to the unique solution $x^{*}$ in $C$ of the variational inequality

$$
\left\langle(I-f) x^{*}, x-x^{*}\right\rangle \geq 0, \quad x \in H .
$$

In [12], Marino and $\mathrm{Xu}$ mixed the iterative method (1.8) and the viscosity approximation method (1.9) and considered the following general iterative method:

$$
x_{n+1}=\left(I-\alpha_{n} A\right) T x_{n}+\alpha_{n} \gamma f\left(x_{n}\right), \quad n \geq 0,
$$

where $A$ is a strongly positive bounded linear operator on $H$. They proved that if the sequence $\left\{\alpha_{n}\right\}$ of parameters satisfies the following conditions

(C1) $\lim _{n \rightarrow \infty} \alpha_{n}=0$,

(C2) $\sum_{n=1}^{\infty} \alpha_{n}=\infty$, and

(C3) $\sum_{n=1}^{\infty}\left|\alpha_{n+1}-\alpha_{n}\right|<\infty$,

then the sequence $\left\{x_{n}\right\}$ generated by (1.11) converges strongly to the unique solution $x^{*}$ in $H$ of the variational inequality

$$
\left\langle(A-\gamma f) x^{*}, x-x^{*}\right\rangle \geq 0, \quad x \in H
$$


which is the optimality condition for the minimization problem: $\min _{x \in C} \frac{1}{2}\langle A x, x\rangle-h(x)$, where $h$ is a potential function for $\gamma f\left(i . e ., h^{\prime}(x)=\gamma f(x)\right.$ for $x \in$ $H)$.

Very recently, Wangkeeree et al. [8] introduced the following general iterative approximation method in the framework of a reflexive Banach space $E$ which admits a weakly continuous duality mapping:

$$
\left\{\begin{array}{l}
x_{0}=x \in E \\
y_{n}=\beta_{n} x_{n}+\left(1-\beta_{n}\right) T_{n} x_{n}, \\
x_{n+1}=\alpha_{n} \gamma f\left(x_{n}\right)+\left(I-\alpha_{n} A\right) y_{n}, \quad n \geq 0,
\end{array}\right.
$$

where $A$ is strongly positive bounded linear operator on $E$ and proved the strong convergence theorems for a countable family of nonexpansive mappings $\left\{T_{n}: E \rightarrow E\right\}_{n=1}^{\infty}$. Other investigations of approximating common fixed points for a countable family of nonexpansive mappings can be found in Refs. $[1,3,8,10-14]$ and many results not cited here.

Inspired and motivated by the iterative (1.13) given above, we give the following modified general iterative scheme for a nonexpansive semigroup $\{T(t): t>0\}$ : for any $\left\{T\left(t_{n}\right): t_{n}>0, n \in \mathbb{N}\right\} \subset\{T(t): t>0\}$,

$$
\left\{\begin{array}{l}
x_{0}=x \in E \\
y_{n}=\beta_{n} x_{n}+\left(1-\beta_{n}\right) T\left(t_{n}\right) x_{n}, \\
x_{n+1}=\alpha_{n} \gamma f\left(x_{n}\right)+\left(I-\alpha_{n} A\right) y_{n}, \quad n \geq 0,
\end{array}\right.
$$

where $\left\{\alpha_{n}\right\},\left\{\beta_{n}\right\}$ and $\left\{t_{n}\right\}$ are real sequence satisfying appropriate control conditions, $A$ is strongly positive bounded linear operator on $E$ and $f$ is a contraction on $E$. The strong convergence theorems are proved in the framework of a reflexive Banach space which admits a weakly continuous duality mapping. Furthermore, by using these results, we obtain strong convergence theorems of the following new iterative schemes $\left\{u_{n}\right\}$ and $\left\{w_{n}\right\}$ defined by

$$
\left\{\begin{array}{l}
u_{0}=u \in E \\
v_{n}=\beta_{n} u_{n}+\left(1-\beta_{n}\right) T\left(t_{n}\right) u_{n} \\
u_{n+1}=\alpha_{n} \gamma f\left(T\left(t_{n}\right) u_{n}\right)+\left(I-\alpha_{n} A\right) v_{n}, \quad n \geq 0
\end{array}\right.
$$

and

$$
\left\{\begin{array}{l}
w_{0}=c \in E \\
v_{n}=\beta_{n} w_{n}+\left(1-\beta_{n}\right) T\left(t_{n}\right) w_{n} \\
w_{n+1}=T\left(t_{n}\right)\left(\alpha_{n} \gamma f\left(w_{n}\right)+\left(I-\alpha_{n} A\right) v_{n}\right), \quad n \geq 0 .
\end{array}\right.
$$

The results presented in this paper improve and extend the corresponding results announced by Marino and $\mathrm{Xu}$ [12], Wangkeeree et al. [8], and Li et al. [15] many others.

\section{Preliminaries}

Throughout this paper, let $E$ be a real Banach space and $E^{*}$ be its dual space. We write $x_{n} \rightarrow x$ (respectively $x_{n} \rightarrow^{*} x$ ) to indicate that the sequence $\left\{x_{n}\right\}$ weakly (respectively weak*) converges to $x$; as usual $x_{n} \rightarrow x$ will symbolize strong convergence. Let $U=\{x$ $\in E:\|x\|=1\}$. A Banach space $E$ is said to uniformly convex if, for any $\varepsilon \in(0,2]$, 
there exists $\delta>0$ such that, for any $x, y \in U,\|x-y\| \geq \varepsilon$ implies $\left\|\frac{x+y}{2}\right\| \leq 1-\delta$. It is known that a uniformly convex Banach space is reflexive and strictly convex (see also [16]). A Banach space $E$ is said to be smooth if the limit $\lim _{t \rightarrow 0} \frac{\|x+t y\|-\|x\|}{t}$ exists for all $x, y \in U$. It is also said to be uniformly smooth if the limit is attained uniformly for $x$, $y \in U$.

Now we collect some useful lemmas for proving the convergence result of this paper.

The first part of the next lemma is an immediate consequence of the subdifferential inequality and the proof of the second part can be found in [17].

Lemma 2.1. ([17]) Assume that a Banach space E has a weakly continuous duality mapping $J_{\phi}$ with gauge $\phi$.

(i) For all $x, y \in E$, the following inequality holds:

$$
\Phi(\|x+y\|) \leq \Phi(\|x\|)+\left\langle y, J_{\varphi}(x+y)\right\rangle .
$$

In particular, for all $x, y \in E$,

$$
\|x+y\|^{2} \leq\|x\|^{2}+2\langle y, J(x+y)\rangle .
$$

(ii) Assume that a sequence $\left\{x_{n}\right\}$ in E converges weakly to a point $x \in E$.

Then the following identity holds:

$$
\limsup _{n \rightarrow \infty} \Phi\left(\left\|x_{n}-y\right\|\right)=\limsup _{n \rightarrow \infty} \Phi\left(\left\|x_{n}-x\right\|\right)+\Phi(\|y-x\|), \quad \forall x, y \in E .
$$

Now, we present the concept of uniformly asymptotically regular semigroup. $\mathcal{S}$ is said to be uniformly asymptotically regular (in short, u.a.r.) on $C$ if for all $h \geq 0$ and any bounded subset $B$ of $C$,

$$
\lim _{s \rightarrow \infty} \sup _{x \in B}\|T(h)(T(s) x)-T(s) x\|=0 .
$$

The nonexpansive semigroup $\left\{\sigma_{t}: t>0\right\}$ defined by the following lemma is an example of u.a.r. nonexpansive semigroup. Other examples of u.a.r. operator semigroup can be found in [[18], Examples 17,18].

Lemma 2.2. (see [[19], Lemma 2.7]). Let $C$ be a nonempty closed convex subset of a uniformly convex Banach space $E, B$ a bounded closed convex subset of $C$, and $\mathcal{S}=\{T(s): 0 \leq s<\infty\}$ a nonexpansive semigroup on $C$ such that $F(\mathcal{S}) \neq \emptyset$. For each $h$ $>0$, set $\sigma_{t}(x)=\frac{1}{t} \int_{0}^{t} T(s) x \mathrm{~d} s$, then

$$
\lim _{t \rightarrow \infty} \sup _{x \in B}\left\|\sigma_{t}(x)-T(h) \sigma_{t}(x)\right\|=0 .
$$

Example 2.3. The set $\left\{\sigma_{t}: t>0\right\}$ defined by Lemma 2.2 is u.a.r. nonexpansive semigroup. In fact, it is obvious that $\left\{\sigma_{t}: t>0\right\}$ is a nonexpansive semigroup. For each $h>$ 0 , we have 


$$
\begin{aligned}
\left\|\sigma_{t}(x)-\sigma_{h} \sigma_{t}(x)\right\| & =\left\|\sigma_{t}(x)-\frac{1}{h} \int_{0}^{h} T(s) \sigma_{t}(x) \mathrm{d} s\right\| \\
& =\left\|\frac{1}{h} \int_{0}^{h}\left(\sigma_{t}(x)-T(s) \sigma_{t}(x)\right) \mathrm{d} s\right\| \\
& \leq \frac{1}{h} \int_{0}^{h}\left\|\sigma_{t}(x)-T(s) \sigma_{t}(x)\right\| \mathrm{d} s .
\end{aligned}
$$

Applying Lemma 2.2, we have

$$
\lim _{t \rightarrow \infty} \sup _{x i n B}\left\|\sigma_{t}(x)-\sigma_{h} \sigma_{t}(x)\right\| \leq \frac{1}{h} \int_{0}^{h} \lim _{t \rightarrow \infty} \sup _{x \in B}\left\|\sigma_{t}(x)-\sigma_{h} \sigma_{t}(x)\right\| \mathrm{d} s=0 .
$$

The next valuable lemma is proved for applying our main results.

Lemma 2.4. [[8], Lemma 3.1] Assume that a Banach space E has a weakly continuous duality mapping $J_{\phi}$ with gauge $\phi$. Let $A$ be a strong positive linear bounded operator on $E$ with coefficient $\bar{\gamma}>0$ and $0<\rho \geq \phi(1)\|A\|^{-1}$. Then $\|I-\rho A\| \leq \varphi(1)(1-\rho \bar{\gamma})$.

Lemma 2.5. ([6]) Assume that $\left\{a_{n}\right\}$ is a sequence of nonnegative real numbers such that

$$
a_{n+1} \leq\left(1-\alpha_{n}\right) a_{n}+b_{n}
$$

where $\left\{\alpha_{n}\right\}$ is a sequence in $(0,1)$ and $\left\{b_{n}\right\}$ is a sequence such that

(a) $\sum_{n=1}^{\infty} \alpha_{n}=\infty$;

(b) $\lim \sup _{n \rightarrow \infty} b_{n} / \alpha_{n} \leq 0$ or $\sum_{n=1}^{\infty}\left\|b_{n}\right\|<\infty$.

Then $\lim _{n \rightarrow \infty} a_{n}=0$.

\section{Main results}

Let $E$ be a Banach space which admits a weakly continuous duality mapping $J_{\phi}$ with gauge $\phi$ such that $\phi$ is invariant on $[0,1]$, i.e., $\phi([0,1]) \subset[0,1]$. Let $\mathcal{S}=\{T(s): s \geq 0\}$ be a nonexpansive semigroups from $C$ into itself. For $f \in \Pi_{E}, t \in(0,1)$, and $A$ is a strongly positive bounded linear operator with coefficient $\bar{\gamma}>0$ and $0<\gamma<\frac{\bar{\gamma} \varphi(1)}{\alpha}$, the mapping $S_{t}: E \rightarrow E$ defined by

$$
S_{t}(x)=t \gamma f(x)+(I-t A) T\left(\lambda_{t}\right) x, \forall x \in E
$$

is a contraction mapping. Indeed, for any $x, y \in E$,

$$
\begin{aligned}
\left\|S_{t}(x)-S_{t}(\gamma)\right\| & =\left\|t \gamma(f(x)-f(\gamma))+(I-t A)\left(T\left(\lambda_{t}\right) x-T\left(\lambda_{t}\right) y\right)\right\| \\
& \leq t \gamma\|f(x)-f(\gamma)\|+\|I-t A\|\left\|T\left(\lambda_{t}\right) x-T\left(\lambda_{t}\right) y\right\| \\
& \leq t \gamma \alpha\|x-\gamma\|+\varphi(1)(1-t \bar{\gamma})\|x-\gamma\| \\
& \leq(1-t(\varphi(1) \bar{\gamma}-\gamma \alpha))\|x-\gamma\| .
\end{aligned}
$$

Thus, by Banach contraction mapping principle, there exists a unique fixed point $x_{t}$ in $E$, that is, 


$$
x_{t}=t \gamma f\left(x_{t}\right)+(I-t A) T\left(\lambda_{t}\right) x_{t} .
$$

Remark 3.1. We note that $l^{p}$ space has a weakly continuous duality mapping with a gauge function $\phi(t)=t^{p-1}$ for all $1<p<\infty$. It is clear that $\phi$ is invariant on $[0,1]$.

Lemma 3.2. Let $E$ be a reflexive Banach space which admits a weakly continuous duality mapping $J_{\phi}$ with gauge $\phi$ such that $\phi$ is invariant on $[0,1]$. Let $\mathcal{S}=\{T(s): s \geq 0\}$ be a nonexpansive semigroup with $F(\mathcal{S}) \neq \emptyset$, and $f \in \Pi_{E}$, let $A$ be a strongly positive bounded linear operator with coefficient $\bar{\gamma}>0$ and $0<\gamma<\frac{\bar{\gamma} \varphi(1)}{\alpha}$, and let $t \in(0,1)$ which satisfying $t \rightarrow 0$. Then the net $\left\{x_{t}\right\}$ defined by (3.2) with $\left\{\lambda_{t}\right\}_{0}<t$ $<1$ is a positive real divergent sequence; converges strongly as $t \rightarrow 0$ to a common fixed point $\tilde{x}$ in $F(\mathcal{S})$ which solves the variational inequality:

$$
\left\langle(A-\gamma f) \tilde{x}_{,} J_{\varphi}(\tilde{x}-z)\right\rangle \leq 0, z \in F(\mathcal{S}) .
$$

Proof. We first show that the uniqueness of a solution of the variational inequality (3.3). Suppose both $\tilde{x} \in F(\mathcal{S})$ and $x^{*} \in F(\mathcal{S})$ are solutions to (3.3), then

$$
\left\langle(A-\gamma f) \tilde{x}, J_{\varphi}\left(\tilde{x}-x^{*}\right)\right\rangle \leq 0
$$

and

$$
\left\langle(A-\gamma f) x^{*}, J_{\varphi}\left(x^{*}-\tilde{x}\right)\right\rangle \leq 0 .
$$

Adding (3.4) and (3.5), we obtain

$$
\left\langle(A-\gamma f) \tilde{x}-(A-\gamma f) x^{*}, J_{\varphi}\left(\tilde{x}-x^{*}\right)\right\rangle \leq 0 .
$$

Noticing that for any $x, y \in E$,

$$
\begin{aligned}
\left\langle(A-\gamma f) x-(A-\gamma f) \gamma_{,} J_{\varphi}(x-\gamma)\right\rangle & =\left\langle A(x-\gamma), J_{\varphi}(x-\gamma)\right\rangle-\gamma\left\langle f(x)-f(\gamma), J_{\varphi}(x-\gamma)\right\rangle \\
& \geq \bar{\gamma}\|x-\gamma\| \varphi(\|x-\gamma\|)-\gamma\|f(x)-f(\gamma)\|\left\|J_{\varphi}(x-\gamma)\right\| \\
& \geq \bar{\gamma} \Phi(\|x-\gamma\|)-\gamma \alpha \Phi(\|x-\gamma\|) \\
& =(\bar{\gamma}-\gamma \alpha) \Phi(\|x-\gamma\|) \\
& \geq(\bar{\gamma} \varphi(1)-\gamma \alpha) \Phi(\|x-\gamma\|) \geq 0 .
\end{aligned}
$$

Using (3.6) and $0<\bar{\gamma} \varphi(1)-\gamma \alpha$ in the last inequality, we get that $\Phi\left(\left\|\tilde{x}-x^{*}\right\|\right)=0$. Therefore, $\tilde{x}=x^{*}$ and the uniqueness is proved. Below we use $\tilde{x}$ to denote the unique solution of (3.3). Next, we will rove that $\left\{x_{t}\right\}$ is bounded. Take a $p \in F(\mathcal{S})$, then we have

$$
\begin{aligned}
\left\|x_{t}-p\right\| & =\left\|t \gamma f\left(x_{t}\right)+(I-t A) T\left(\lambda_{t}\right) x_{t}-p\right\| \\
& =\left\|(I-t A) T\left(\lambda_{t}\right) x_{t}-(I-t A) p+t\left(\gamma f\left(x_{t}\right)-A(p)\right)\right\| \\
& \leq\|\varphi(1)(1-t \bar{\gamma})\| x_{t}-p \|+t\left(\gamma \alpha\left\|x_{t}-p\right\|+\|\gamma f(p)-A(p)\|\right) .
\end{aligned}
$$

It follows that

$$
\left\|x_{t}-p\right\| \leq \frac{1}{\bar{\gamma} \varphi(1)-\gamma \alpha}\|\gamma f(p)-A(p)\| .
$$

Hence, $\left\{x_{t}\right\}$ is bounded, so are $\left\{f\left(x_{t}\right)\right\}$ and $\left\{A T\left(x_{t}\right)\right\}$. The definition of $\left\{x_{t}\right\}$ implies that

$$
\left\|x_{t}-T\left(\lambda_{t}\right) x_{t}\right\|=t\left\|\gamma f\left(x_{t}\right)-A\left(T\left(\lambda_{t}\right) x_{t}\right)\right\| \rightarrow 0 \text { as } t \rightarrow 0 .
$$


Next, we show that $\left\|x_{t}-T(h) x_{t}\right\| \rightarrow 0$ for all $h \geq 0$. Since $\{T(t): t \geq 0\}$ is u.a.r. nonexpansive semigroup and $\lim _{t \rightarrow 0} \lambda_{t}=\infty$, then, for all $h>0$ and for any bounded subset $D$ of $C$ containing $\left\{x_{t}\right\}$,

$$
\lim _{t \rightarrow 0}\left\|T(h)\left(T\left(\lambda_{t}\right) x_{t}\right)-T\left(\lambda_{t}\right) x_{t}\right\| \leq \lim _{t \rightarrow 0} \sup _{x \in D}\left\|T(h)\left(T\left(\lambda_{t}\right) x_{t}\right)-T\left(\lambda_{t}\right) x_{t}\right\|=0 .
$$

Hence, when $t \rightarrow 0$, for all $h>0$, we have

$$
\begin{aligned}
\left\|x_{t}-T(h) x_{t}\right\| & \leq\left\|x_{t}-T\left(\lambda_{t}\right) x_{t}\right\|+\left\|T\left(\lambda_{t}\right) x_{t}-T(h)\left(T(\lambda) x_{t}\right)\right\|+\left\|T(h)\left(T\left(\lambda_{t}\right) x_{t}\right)-T(h) x_{t}\right\| \\
& \leq 2\left\|x_{t}-T\left(\lambda_{t}\right) x_{t}\right\|+\left\|T\left(\lambda_{t}\right) x_{t}-T(h)\left(T\left(\lambda_{t}\right) x_{t}\right)\right\| \rightarrow 0 .
\end{aligned}
$$

Assume that $\left\{t_{n}\right\}_{n=1}^{\infty} \subset(0,1)$ is such that $t_{n} \rightarrow 0$ as $n \rightarrow \infty$. Put $x_{n}:=x_{t_{n}}$ and $\lambda_{n}:=\lambda_{t_{n}}$. We show that $\left\{x_{n}\right\}$ contains a subsequence converging strongly to $\tilde{x} \in F(\mathcal{S})$. It follows from reflexivity of $E$ and the boundedness of sequence $\left\{x_{n}\right\}$ that there exists $\left\{x_{n_{j}}\right\}$ which is a subsequence of $\left\{x_{n}\right\}$ converging weakly to $w \in E$ as $n \rightarrow \infty$. Since $J_{\phi}$ is weakly sequentially continuous, we have by Lemma 2.1 that

$$
\limsup _{j \rightarrow \infty} \Phi\left(\left\|x_{n_{j}}-x\right\|\right)=\limsup _{j \rightarrow \infty} \Phi\left(\left\|x_{n_{j}}-w\right\|\right)+\Phi(\|x-w\|), \quad \text { for all } x \in E .
$$

Let

$$
H(x)=\limsup _{j \rightarrow \infty} \Phi\left(\left\|x_{n_{j}}-x\right\|\right), \quad \text { for all } x \in E .
$$

It follows that

$$
H(x)=H(w)+\Phi(\|x-w\|), \quad \text { for all } x \in E .
$$

For $h \geq 0$, from (3.9) we obtain

$$
\begin{aligned}
H(T(h) w) & =\limsup _{j \rightarrow \infty} \Phi\left(\left\|x_{n_{j}}-T(h) w\right\|\right)=\limsup _{j \rightarrow \infty} \Phi\left(\left\|T(h) x_{n_{j}}-T(h) w\right\|\right) \\
& \leq \limsup _{j \rightarrow \infty} \Phi\left(\left\|x_{n_{j}}-w\right\|\right)=H(w) .
\end{aligned}
$$

On the other hand, however,

$$
H(T(h) w)=H(w)+\Phi(\|T(h) w-w\|) .
$$

It follows from (3.10) and (3.11) that

$$
\Phi(\|T(h) w-w\|)=H(T(h) w)-H(w) \leq 0 .
$$

This implies that $T(h) w=w$ for all $h \geq 0$, and so $w \in F(\mathcal{S})$. Next, we show that $x_{n_{j}} \rightarrow w$ as $j \rightarrow \infty$. In fact, since $\Phi(t)=\int_{0}^{t} \varphi(\tau) \mathrm{d} \tau, \forall t \geq 0$, and $\phi:[0, \infty) \rightarrow[0, \infty)$ is a gauge function, then for $1 \geq k \geq 0, \phi(k x) \leq \phi(x)$ and

$$
\Phi(k t)=\int_{0}^{k t} \varphi(\tau) \mathrm{d} \tau=k \int_{0}^{t} \varphi(k x) \mathrm{d} x \leq k \int_{0}^{t} \varphi(x) \mathrm{d} x=k \Phi(t) .
$$


Following Lemma 2.1, we have

$$
\begin{aligned}
\Phi\left(\left\|x_{n}-w\right\|\right)= & \Phi\left(\left(I-t_{n} A\right) T\left(t_{n}\right) x_{n}-\left(I-t_{n} A\right) w+t_{n}\left(\gamma f\left(x_{n}\right)-A(w)\right)\right) \\
= & \Phi\left(\left\|\left(I-t_{n} A\right) T\left(t_{n}\right) x_{n}-\left(I-t_{n} A\right) w\right\|\right)+t_{n}\left\langle\gamma f\left(x_{n}\right)-A(w), J_{\varphi}\left(x_{n}-w\right)\right\rangle \\
\leq & \Phi\left(\varphi(1)\left(1-t_{n} \bar{\gamma}\right)\left\|x_{n}-w\right\|\right)+t_{n} \gamma\left\langle f\left(x_{t_{n}}\right)-f(w), J_{\varphi}\left(x_{n}-w\right)\right\rangle \\
& +t_{n}\left\langle\gamma f(w)-A(w), J_{\varphi}\left(x_{n}-w\right)\right\rangle \\
\leq & \varphi(1)\left(1-t_{n} \bar{\gamma}\right) \Phi\left(\left\|x_{n}-w\right\|\right)+t_{n} \gamma\left\|f\left(x_{n}\right)-f(w)\right\|\left\|J_{\varphi}\left(x_{n}-w\right)\right\| \\
& +t_{n}\left\langle\gamma f(w)-A(w), J_{\varphi}\left(x_{n}-w\right)\right\rangle \\
\leq & \varphi(1)\left(1-t_{n} \bar{\gamma}\right) \Phi\left(\left\|x_{n}-w\right\|\right)+t_{n} \gamma \alpha\left\|x_{n}-w\right\|\left\|J_{\varphi}\left(x_{n}-w\right)\right\| \\
& +t_{n}\left\langle\gamma f(w)-A(w), J_{\varphi}\left(x_{n}-w\right)\right\rangle \\
= & \varphi(1)\left(1-t_{n} \bar{\gamma}\right) \Phi\left(\left\|x_{n}-w\right\|\right)+t_{n} \gamma \alpha \Phi\left(\left\|x_{n}-w\right\|\right) \\
& +t_{n}\left\langle\gamma f(w)-A(w), J_{\varphi}\left(x_{n}-w\right)\right\rangle \\
= & \left(1-t_{n}(\bar{\gamma} \varphi(1)-\gamma \alpha)\right) \Phi\left(\left\|x_{n}-w\right\|\right)+t_{n}\left\langle\gamma f(w)-A(w), J_{\varphi}\left(x_{n}-w\right)\right\rangle .
\end{aligned}
$$

This implies that

$$
\Phi\left(\left\|x_{n_{j}}-w\right\|\right) \leq \frac{1}{\bar{\gamma} \varphi(1)-\gamma \alpha}\left\langle\gamma f(w)-A(w), J_{\varphi}\left(x_{n_{j}}-w\right)\right\rangle .
$$

Now observing that $x_{n} \rightarrow w$ implies $J_{\phi}\left(x_{n}-w\right) \rightarrow 0$, we conclude from the last inequality that

$$
\Phi\left(\left\|x_{n_{j}}-w\right\|\right) \rightarrow 0 \text { as } j \rightarrow \infty .
$$

Hence, $x_{n_{j}} \rightarrow w$ as $j \rightarrow \infty$. Next, we prove that $w$ solves the variational inequality (3.3). For any $z \in F(\mathcal{S})$, we observe that

$$
\begin{aligned}
\left\langle\left(I-T\left(\lambda_{t}\right)\right) x_{t}-\left(I-T\left(\lambda_{t}\right)\right) z_{1} J_{\varphi}\left(x_{t}-z\right)\right\rangle & =\left\langle x_{t}-z_{1} J_{\varphi}\left(x_{t}-z\right)\right\rangle+\left\langle T\left(\lambda_{t}\right) x_{t}-T\left(\lambda_{t}\right) z_{1} J_{\varphi}\left(x_{t}-z\right)\right\rangle \\
& =\Phi\left(\left\|x_{t}-z\right\|\right)-\left\langle T\left(\lambda_{t}\right) z-T\left(\lambda_{t}\right) x_{t}, J_{\varphi}\left(x_{t}-z\right)\right\rangle \\
& \geq \Phi\left(\left\|x_{t}-z\right\|\right)-\left\|T\left(\lambda_{t}\right) z-T\left(\lambda_{t}\right) x_{t}\right\|\left\|J_{\varphi}\left(x_{t}-z\right)\right\| \\
& \geq \Phi\left(\left\|x_{t}-z\right\|\right)-\left\|z-x_{t}\right\|\left\|J_{\varphi}\left(x_{t}-z\right)\right\| \\
& =\Phi\left(\left\|x_{t}-z\right\|\right)-\Phi\left(\left\|x_{t}-z\right\|\right)=0 .
\end{aligned}
$$

Since

$$
x_{t}=t \gamma f\left(x_{t}\right)+(I-t A) T\left(\lambda_{t}\right) x_{t}
$$

we can derive that

$$
(A-\gamma f)\left(x_{t}\right)=-\frac{1}{t}\left(I-T\left(\lambda_{t}\right)\right) x_{t}+\left(A\left(I-T\left(\lambda_{t}\right)\right) x_{t}\right) .
$$

Thus,

$$
\begin{aligned}
\left\langle(A-\gamma f)\left(x_{t}\right), J_{\varphi}\left(x_{t}-z\right)\right\rangle & =-\frac{1}{t}\left\langle\left(I-T\left(\lambda_{t}\right)\right) x_{t}-\left(I-T\left(\lambda_{t}\right)\right) z_{1} J_{\varphi}\left(x_{t}-z\right)\right\rangle+\left\langle A\left(I-T\left(\lambda_{t}\right)\right) x_{t}, J_{\varphi}\left(x_{t}-z\right)\right\rangle \\
& \leq\left\langle A\left(I-T\left(\lambda_{t}\right)\right) x_{t}, J_{\varphi}\left(x_{t}-z\right)\right\rangle .
\end{aligned}
$$

Noticing that

$$
x_{n_{j}}-T\left(\lambda_{t_{n_{j}}}\right) x_{n_{j}} \rightarrow 0 .
$$

Now replacing $t$ and $\lambda_{t}$ with $n_{j}$ and $t_{n_{j}}$ in (3.14) and letting $j \rightarrow \infty$, we have

$$
\left\langle(A-\gamma f) w, J_{\varphi}(w-z)\right\rangle \leq 0 \text {. }
$$


So, $w \in F(T)$ is a solution of the variational inequality (3.3), and hence, $w=\tilde{x}$ by the uniqueness. In a summary, we have shown that each cluster point of $\left\{x_{t}\right\}$ (at $\left.t \rightarrow 0\right)$ equals $\tilde{x}$. Therefore, $x_{t} \rightarrow \tilde{x}$ as $t \rightarrow 0$. This completes the proof.

Theorem 3.3. Let $E$ be a reflexive Banach space which admits a weakly continuous duality mapping $J_{\phi}$ with gauge $\phi$ such that $\phi$ is invariant on $[0,1]$. Let $\{T(s): s \geq 0\}$ be a u.a.r. semigroup of nonexpansive mappings with $F(\mathcal{S}) \neq \emptyset$, and $f \in \Pi_{E}$, let $A$ be a strongly positive bounded linear operator with coefficient $\bar{\gamma}>0$ and $0<\gamma<\frac{\bar{\gamma} \varphi(1)}{\alpha}$. Let the sequence $\left\{x_{n}\right\}$ be generated by the following:

$$
\left\{\begin{array}{l}
x_{0}=x \in E \\
y_{n}=\beta_{n} x_{n}+\left(1-\beta_{n}\right) T\left(t_{n}\right) x_{n} \\
x_{n+1}=\alpha_{n} \gamma f\left(x_{n}\right)+\left(I-\alpha_{n} A\right) y_{n}, \quad n \geq 0
\end{array}\right.
$$

where $\left\{\alpha_{n}\right\} \subset(0,1)$ and $\left\{\beta_{n}\right\} \subset[0,1]$ are real sequences satisfying the following conditions:

(C1) $\lim _{n \rightarrow \infty} \alpha_{n}=0$ and $\sum_{n=1}^{\infty} \alpha_{n}=\infty$

(C2) $\lim _{n \rightarrow \infty} \beta_{n}=0$,

(C3) $\lim _{n \rightarrow \infty} t_{n}=\infty$.

Then $\left\{x_{n}\right\}$ converges strongly to $\tilde{x}$ that is obtained in Lemma 3.2.

Proof. Since $\lim _{n \rightarrow \infty} \alpha_{n}=0$, we may assume, without loss of generality, that $\alpha_{n}<\phi$ (1) $\|A\|^{-1}$ for all $n$. By Lemma 2.4 , we have $\left\|I-\alpha_{n} A\right\| \leq \varphi(1)\left(1-\alpha_{n} \bar{\gamma}\right)$. We first observe that $\left\{x_{n}\right\}$ is bounded. Indeed, pick any $p \in F(\mathcal{S})$ to obtain

$$
\begin{aligned}
\left\|y_{n}-p\right\| & =\left\|\beta_{n} x_{n}+\left(1-\beta_{n}\right) T\left(t_{n}\right) x_{n}-p\right\| \\
& =\left\|\beta_{n}\left(x_{n}-p\right)+\left(1-\beta_{n}\right)\left(T\left(t_{n}\right) x_{n}-T\left(t_{n}\right) p\right)\right\| \\
& \leq \beta_{n}\left\|x_{n}-p\right\|+\left(1-\beta_{n}\right)\left\|x_{n}-p\right\| \\
& =\left\|x_{n}-p\right\|
\end{aligned}
$$

and so

$$
\begin{aligned}
\left\|x_{n+1}-p\right\| & =\left\|\alpha_{n} \gamma f\left(x_{n}\right)+\left(I-\alpha_{n} A\right) y_{n}-p\right\| \\
& =\left\|\alpha_{n}\left(\gamma f\left(x_{n}\right)-A(p)\right)+\left(I-\alpha_{n} A\right) y_{n}-\left(I-\alpha_{n} A\right) p\right\| \\
& \leq \alpha_{n}\left\|\gamma f\left(x_{n}\right)-A(p)\right\|+\varphi(1)\left(1-\alpha_{n} \bar{\gamma}\right)\left\|y_{n}-p\right\| \\
& \leq \alpha_{n} \gamma\left\|f\left(x_{n}\right)-f(p)\right\|+\alpha_{n}\|\gamma f(p)-A(p)\|+\varphi(1)\left(1-\alpha_{n} \bar{\gamma}\right)\left\|y_{n}-p\right\| \\
& \leq \alpha_{n} \gamma \alpha\left\|x_{n}-p\right\|+\alpha_{n}\|\gamma f(p)-A(p)\|+\varphi(1)\left(1-\alpha_{n} \bar{\gamma}\right)\left\|x_{n}-p\right\| \\
& \leq\left(1-\alpha_{n}(\bar{\gamma} \varphi(1)-\gamma \alpha)\right)\left\|x_{n}-p\right\|+\alpha_{n}\left\|\gamma f\left(x_{n}\right)-A(p)\right\| \\
& =\left(1-\alpha_{n}(\bar{\gamma} \varphi(1)-\gamma \alpha)\right)\left\|x_{n}-p\right\|+\alpha_{n}(\bar{\gamma} \varphi(1)-\gamma \alpha) \frac{\left\|\gamma f\left(x_{n}\right)-A(p)\right\|}{\bar{\gamma} \varphi(1)-\gamma \alpha} .
\end{aligned}
$$

It follows from induction that

$$
\left\|x_{n}-p\right\| \leq \max \left\{\left\|x_{0}-p\right\|, \frac{\|\gamma f(p)-A(p)\|}{\bar{\gamma} \varphi(1)-\gamma \alpha}\right\}, \quad n \geq 0 .
$$

The boundedness of $\left\{x_{n}\right\}$ implies that $\left\{y_{n}\right\},\left\{T\left(t_{n}\right) x_{n}\right\}$ and $\left\{f\left(x_{n}\right)\right\}$ are bounded. 
Thus by (3.29), (C1) and (C2), we have

$$
\left\|y_{n}-T\left(t_{n}\right) x_{n}\right\|=\beta_{n}\left\|x_{n}-T\left(t_{n}\right) x_{n}\right\| \rightarrow 0
$$

and there by,

$$
\left\|x_{n+1}-T\left(t_{n}\right) x_{n}\right\| \leq\left\|y_{n}-T\left(t_{n}\right) x_{n}\right\|+\alpha_{n}\left\|\gamma f\left(x_{n}\right)-A\left(y_{n}\right)\right\| \rightarrow 0 .
$$

Since $\{T(t): t \geq 0\}$ is u.a.r. nonexpansive semigroup and $\lim _{n \rightarrow \infty} t_{n}=\infty$, then, for all $h$ $>0$ and for any bounded subset $D$ of $C$ containing $\left\{x_{n}\right\}$,

$$
\lim _{n \rightarrow \infty}\left\|T(h)\left(T\left(t_{n}\right) x_{n}\right)-T\left(t_{n}\right) x_{n}\right\| \leq \lim _{n \rightarrow \infty} \sup _{x \in D}\left\|T(h)\left(T\left(t_{n}\right) x_{n}\right)-T\left(t_{n}\right) x_{n}\right\|=0 .
$$

Hence, when $n \rightarrow \infty$, for all $h>0$, we have

$$
\begin{aligned}
\left\|x_{n+1}-T(h) x_{n+1}\right\| & \leq\left\|x_{n+1}-T\left(t_{n}\right) x_{n}\right\|+\left\|T\left(t_{n}\right) x_{n}-T(h)\left(T\left(t_{n}\right) x_{n}\right)\right\|+\left\|T(h)\left(T\left(t_{n}\right) x_{n}\right)-T(h) x_{n+1}\right\| \\
& \leq 2\left\|x_{n+1}-T\left(t_{n}\right) x_{n}\right\|+\left\|T\left(t_{n}\right) x_{n}-T(h)\left(T\left(t_{n}\right) x_{n}\right)\right\| \rightarrow 0
\end{aligned}
$$

Next, we prove that

$$
\limsup _{n \rightarrow \infty}\left\langle\gamma f(\tilde{x})-A \tilde{x}, J_{\varphi}\left(x_{n}-\tilde{x}\right)\right\rangle \leq 0,
$$

Let $\left\{x_{n_{k}}\right\}$ be a subsequence of $\left\{x_{n}\right\}$ such that

$$
\lim _{k \rightarrow \infty}\left\langle\gamma f(\tilde{x})-A \tilde{x}, J_{\varphi}\left(x_{n_{k}}-\tilde{x}\right)\right\rangle=\limsup _{n \rightarrow \infty}\left\langle\gamma f(\tilde{x})-A \tilde{x}, J_{\varphi}\left(x_{n}-\tilde{x}\right)\right\rangle .
$$

If follows from reflexivity of $E$ and the boundedness of sequence $\left\{x_{n_{k}}\right\}$ that there exists $\left\{x_{n_{k_{i}}}\right\}$ which is a subsequence of $\left\{x_{n_{k}}\right\}$ converging weakly to $w \in E$ as $i \rightarrow \infty$. Since $J_{\phi}$ is weakly continuous, we have by Lemma 2.1 that

$$
\limsup _{n \rightarrow \infty} \Phi\left(\left\|x_{n_{k_{i}}}-x\right\|\right)=\limsup _{n \rightarrow \infty} \Phi\left(\left\|x_{n_{k_{i}}}-w\right\|\right)+\Phi(\|x-w\|), \quad \text { for all } x \in E .
$$

Let

$$
H(x)=\limsup _{n \rightarrow \infty} \Phi\left(\left\|x_{n_{k_{i}}}-x\right\|\right), \quad \text { for all } x \in E .
$$

It follows that

$$
H(x)=H(w)+\Phi(\|x-w\|), \quad \text { for all } x \in E .
$$

From (3.18), for each $h>0$, we obtain

$$
\begin{aligned}
H(T(h) w) & =\limsup _{i \rightarrow \infty} \Phi\left(\left\|x_{n_{k_{i}}}-T(h) w\right\|\right)=\limsup _{i \rightarrow \infty} \Phi\left(\left\|T(h) x_{n_{k_{i}}}-T(h) w\right\|\right) \\
& \leq \limsup _{i \rightarrow \infty} \Phi\left(\left\|x_{n_{k_{i}}}-w\right\|\right)=H(w)
\end{aligned}
$$

On the other hand, however,

$$
H(T(h) w)=H(w)+\Phi(\|T(h) w-w\|)
$$

It follows from (3.21) and (3.22) that

$$
\Phi(\|T(h) w-w\|)=H(T(h) w)-H(w) \leq 0 .
$$

This implies that $T(h) w=w$ for all $h>0$, and so $w \in F(\mathcal{S})$. Since the duality map $J_{\phi}$ is single-valued and weakly continuous, we get that 


$$
\begin{aligned}
\limsup _{n \rightarrow \infty}\left\langle\gamma f(\tilde{x})-A \tilde{x}, J_{\varphi}\left(x_{n}-\tilde{x}\right)\right\rangle & =\lim _{k \rightarrow \infty}\left\langle\gamma f(\tilde{x})-A \tilde{x}, J_{\varphi}\left(x_{n_{k}}-\tilde{x}\right)\right\rangle \\
& =\lim _{i \rightarrow \infty}\left\langle\gamma f(\tilde{x})-A \tilde{x}, J_{\varphi}\left(x_{n_{k_{i}}}-\tilde{x}\right)\right\rangle \\
& =\left\langle(A-\gamma f) \tilde{x}, J_{\varphi}(\tilde{x}-w)\right\rangle \leq 0
\end{aligned}
$$

as required.

Finally, we show that $x_{n} \rightarrow \tilde{x}$ as $n \rightarrow \infty$.

$$
\begin{aligned}
\Phi\left(\left\|x_{n+1}-\tilde{x}\right\|\right) & =\Phi\left(\left\|\alpha_{n}\left(\gamma f\left(x_{n}\right)\right)+\left(I-\alpha_{n} A\right) y_{n}-\tilde{x}\right\|\right) \\
& =\Phi\left(\left\|\alpha_{n}\left(\gamma f\left(x_{n}\right)-A \tilde{x}\right)+\left(I-\alpha_{n} A\right)\left(y_{n}-\tilde{x}\right)\right\|\right) \\
& =\Phi\left(\left\|\alpha_{n}\left(\gamma f\left(x_{n}\right)-\gamma f(\tilde{x})\right)+\alpha_{n}(\gamma f(\tilde{x})-A \tilde{x})+\left(I-\alpha_{n} A\right)\left(y_{n}-\tilde{x}\right)\right\|\right) \\
& \leq \Phi\left(\left\|\alpha_{n}\left(\gamma f\left(x_{n}\right)-\gamma f(\tilde{x})\right)+\left(I-\alpha_{n} A\right)\left(y_{n}-\tilde{x}\right)\right\|\right)+\alpha_{n}\left\langle\gamma f(\tilde{x})-A \tilde{x}, J_{\varphi}\left(x_{n+1}-\tilde{x}\right)\right\rangle \\
& \leq \Phi\left(\left\|\alpha_{n}\left(\gamma f\left(x_{n}\right)-\gamma f(\tilde{x})\right)\right\|+\left\|\left(I-\alpha_{n} A\right)\left(y_{n}-\tilde{x}\right)\right\|\right)+\alpha_{n}\left\langle\gamma f(\tilde{x})-A \tilde{x}, J_{\varphi}\left(x_{n+1}-\tilde{x}\right)\right\rangle \\
& \left.\leq \Phi\left(\alpha_{n} \gamma \alpha\left\|x_{n}-\tilde{x}\right\|+\varphi(1)\left(1-\alpha_{n} \bar{\gamma}\right)\left\|y_{n}-\tilde{x}\right\|\right)\right)+\alpha_{n}\left\langle\gamma f(\tilde{x})-A \tilde{x}, J_{\varphi}\left(x_{n+1}-\tilde{x}\right)\right\rangle \\
& \left.\leq \Phi\left(\alpha_{n} \gamma \alpha\left\|x_{n}-\tilde{x}\right\|+\varphi(1)\left(1-\alpha_{n} \bar{\gamma}\right)\left\|x_{n}-\tilde{x}\right\|\right)\right)+\alpha_{n}\left\langle\gamma f(\tilde{x})-A \tilde{x}_{,} J_{\varphi}\left(x_{n+1}-\tilde{x}\right)\right\rangle \\
& =\Phi\left(\left(\varphi(1)-\alpha_{n}(\varphi(1) \bar{\gamma}-\gamma \alpha)\right)\left\|x_{n}-\tilde{x}\right\|\right)+\alpha_{n}\left\langle\gamma f(\tilde{x})-A \tilde{x}, J_{\varphi}\left(x_{n+1}-\tilde{x}\right)\right\rangle \\
& \left.\leq\left(1-\alpha_{n}(\varphi(1) \bar{\gamma}-\gamma \alpha)\right) \Phi\left(\left\|x_{n}-\tilde{x}\right\|\right)\right)+\alpha_{n}\left\langle\gamma f(\tilde{x})-A \tilde{x}, J_{\varphi}\left(x_{n+1}-\tilde{x}\right)\right\rangle .
\end{aligned}
$$

Apply Lemma 2.5 to (3.23) to conclude $\Phi\left(\left\|x_{n+1}-\tilde{x}\right\|\right) \rightarrow 0$ as $n \rightarrow \infty$, that is, $x_{n} \rightarrow \tilde{x}$ as $n \rightarrow \infty$. This completes the proof.

Corollary 3.4. Let $E$ be a reflexive Banach space which admits a weakly continuous duality mapping $J_{\phi}$ with gauge $\phi$ such that $\phi$ is invariant on $[0,1]$. Let $\{T(s): s \geq 0\}$ be a u.a.r. semigroup of nonexpansive mappings with $F(\mathcal{S}) \neq \emptyset$, and $f \in \Pi_{E}$, let $A$ be a strongly positive bounded linear operator with coefficient $\bar{\gamma}>0$ and $0<\gamma<\frac{\bar{\gamma} \varphi(1)}{\alpha}$. Let the sequence $\left\{x_{n}\right\}$ be generated by the following:

$$
\left\{\begin{array}{l}
u_{0}=u \in E \\
v_{n}=\beta_{n} u_{n}+\left(1-\beta_{n}\right) T\left(t_{n}\right) u_{n}, \\
u_{n+1}=\alpha_{n} \gamma f\left(T\left(t_{n}\right) u_{n}\right)+\left(I-\alpha_{n} A\right) v_{n}, \quad n \geq 0
\end{array}\right.
$$

where $\left\{\alpha_{n}\right\} \subset(0,1)$ and $\left\{\beta_{n}\right\} \subset[0,1]$ are real sequences satisfying the following conditions:

(C1) $\lim _{n \rightarrow \infty} \alpha_{n}=0$ and $\sum_{n=1}^{\infty} \alpha_{n}=\infty$

(C2) $\lim _{n \rightarrow \infty} \beta_{n}=0$,

(C3) $\lim _{n \rightarrow \infty} t_{n}=\infty$.

Then $\left\{u_{n}\right\}$ converges strongly to $\tilde{x}$ that is obtained in Lemma 3.2.

Proof. Let $\left\{x_{n}\right\}$ be the sequence in given by $x_{0}=u_{0}$ and

$$
\left\{\begin{array}{l}
y_{n}=\beta_{n} x_{n}+\left(1-\beta_{n}\right) T\left(t_{n}\right) x_{n} \\
x_{n+1}=\alpha_{n} \gamma f\left(x_{n}\right)+\left(I-\alpha_{n} A\right) y_{n}, \quad n \geq 0
\end{array}\right.
$$

From Theorem 3.3, $x_{n} \rightarrow \tilde{x}$. We claim that $u_{n} \rightarrow \tilde{x}$. From (3.26) and (3.25), we have

$$
\begin{aligned}
\left\|y_{n}-v_{n}\right\| & =\left\|\beta_{n} x_{n}+\left(1-\beta_{n}\right) T\left(t_{n}\right) x_{n}-\beta_{n} u_{n}-\left(1-\beta_{n}\right) T\left(t_{n}\right) u_{n}\right\| \\
& \leq \beta_{n}\left\|x_{n}-u_{n}\right\|+\left(1-\beta_{n}\right)\left\|T\left(t_{n}\right) x_{n}-T\left(t_{n}\right) u_{n}\right\| \\
& \leq \beta_{n}\left\|x_{n}-u_{n}\right\|+\left(1-\beta_{n}\right)\left\|x_{n}-u_{n}\right\| \\
& =\left\|x_{n}-u_{n}\right\| .
\end{aligned}
$$


Again, it then follows that

$$
\begin{aligned}
\left\|x_{n+1}-u_{n+1}\right\| & =\left\|\alpha_{n} \gamma f\left(x_{n}\right)+\left(I-\alpha_{n} A\right) y_{n}-\alpha_{n} \gamma f\left(T\left(t_{n}\right) u_{n}\right)-\left(I-\alpha_{n} A\right) v_{n}\right\| \\
& \leq \alpha_{n} \gamma\left\|f\left(x_{n}\right)-f\left(T\left(t_{n}\right) u_{n}\right)\right\|+\left\|I-\alpha_{n} A\right\|\left\|y_{n}-v_{n}\right\| \\
& \leq \alpha_{n} \gamma \alpha\left\|x_{n}-T\left(t_{n}\right) u_{n}\right\|+\varphi(1)\left(1-\alpha_{n} \bar{\gamma}\right)\left\|x_{n}-u_{n}\right\| \\
& \leq \alpha_{n} \gamma \alpha\left\|x_{n}-T\left(t_{n}\right) \tilde{x}\right\|+\alpha_{n} \gamma \alpha\left\|T\left(t_{n}\right) \tilde{x}-T\left(t_{n}\right) u_{n}\right\|+\varphi(1)\left(1-\alpha_{n} \bar{\gamma}\right)\left\|x_{n}-u_{n}\right\| \\
& \leq \alpha_{n} \gamma \alpha\left\|x_{n}-\tilde{x}\right\|+\alpha_{n} \gamma \alpha\left\|\tilde{x}-u_{n}\right\|+\varphi(1)\left(1-\alpha_{n} \bar{\gamma}\right)\left\|x_{n}-u_{n}\right\| \\
& =\alpha_{n} \gamma \alpha\left\|x_{n}-\tilde{x}\right\|+\alpha_{n} \gamma \alpha\left\|\tilde{x}-x_{n}\right\|+\alpha_{n} \gamma \alpha\left\|x_{n}-u_{n}\right\|+\varphi(1)\left(1-\alpha_{n} \bar{\gamma}\right)\left\|x_{n}-u_{n}\right\| \\
& =\left(\varphi(1)\left(1-\alpha_{n} \bar{\gamma}\right)+\alpha_{n} \gamma \alpha\right)\left\|x_{n}-u_{n}\right\|+\left(\alpha_{n} \gamma \alpha+\alpha_{n} \gamma \alpha\right)\left\|x_{n}-\tilde{x}\right\| \\
& \leq\left(1-\alpha_{n}(\varphi(1) \bar{\gamma}-\gamma \alpha)\right)\left\|x_{n}-u_{n}\right\|+\alpha_{n}(\varphi(1) \bar{\gamma}-\gamma \alpha) \frac{2 \gamma \alpha}{(\varphi(1) \bar{\gamma}-\gamma \alpha)}\left\|x_{n}-\tilde{x}\right\| .
\end{aligned}
$$

It follows from $\sum_{n=1}^{\infty} \alpha_{n}=\infty, \lim _{n \rightarrow \infty}\left\|x_{n}-\tilde{x}\right\|=0$, and Lemma 2.5 that $\left\|x_{n}-u_{n}\right\|$ $\rightarrow 0$. Consequently, $u_{n} \rightarrow \tilde{x}$ as required.

Corollary 3.5. Let E be a reflexive Banach space which admits a weakly continuous duality mapping $J_{\phi}$ with gauge $\phi$ such that $\phi$ is invariant on $[0,1]$. Let $\{T(s): s \geq 0\}$ be a u.a.r. semigroup of nonexpansive mappings with $F(\mathcal{S}) \neq \emptyset$, and $f \in \Pi_{E}$, let $A$ be a strongly positive bounded linear operator with coefficient $\bar{\gamma}>0$ and $0<\gamma<\frac{\bar{\gamma} \varphi(1)}{\alpha}$. Let the sequence $\left\{x_{n}\right\}$ be generated by the following:

$$
\left\{\begin{array}{l}
w_{0}=w \in E \\
v_{n}=\beta_{n} w_{n}+\left(1-\beta_{n}\right) T\left(t_{n}\right) w_{n}, \\
w_{n+1}=T\left(t_{n}\right)\left(\alpha_{n} \gamma f\left(w_{n}\right)+\left(I-\alpha_{n} A\right) v_{n}\right), \quad n \geq 0
\end{array}\right.
$$

where $\left\{\alpha_{n}\right\} \subset(0,1)$ and $\left\{\beta_{n}\right\} \subset[0,1]$ are real sequences satisfying the following conditions:

(C1) $\lim _{n \rightarrow \infty} \alpha_{n}=0$ and $\sum_{n=1}^{\infty} \alpha_{n}=\infty$

(C2) $\lim _{n \rightarrow \infty} \beta_{n}=0$,

(C3) $\lim _{n \rightarrow \infty} t_{n}=\infty$.

Then $\left\{w_{n}\right\}$ converges strongly to $\tilde{x}$ that is obtained in Lemma 3.2.

Proof. Define the sequence $\left\{u_{n}\right\}$ and $\left\{\sigma_{n}\right\}$ by

$$
u_{n}=\alpha_{n} \gamma f\left(w_{n}\right)+\left(I-\alpha_{n} A\right) w_{n}, \sigma_{n}=\alpha_{n+1} \quad \forall n \geq 0 .
$$

Taking $p \in F(\mathcal{S})$, we have

$$
\begin{aligned}
\left\|w_{n+1}-p\right\| & =\left\|T\left(t_{n}\right) u_{n}-T\left(t_{n}\right) p\right\| \leq\left\|u_{n}-p\right\| \\
& =\left\|\alpha_{n} \gamma f\left(w_{n}\right)+\left(I-\alpha_{n} A\right) w_{n}-\left(I-\alpha_{n} A\right) p-\alpha_{n} A p\right\| \\
& \leq \alpha_{n}\left\|\gamma f\left(w_{n}\right)-A p\right\|+\left\|I-\alpha_{n} A\right\|\left\|w_{n}-p\right\| \\
& \leq \alpha_{n}\left\|\gamma f\left(w_{n}\right)-A p\right\|+\varphi(1)\left(1-\alpha_{n} \bar{\gamma}\right)\left\|w_{n}-p\right\| \\
& \leq \alpha_{n}\left\|\gamma f\left(w_{n}\right)-\gamma f(p)\right\|+\alpha_{n}\|\gamma f(p)-A p\|+\varphi(1)\left(1-\alpha_{n} \bar{\gamma}\right)\left\|w_{n}-p\right\| \\
& \leq \alpha_{n} \gamma \alpha\left\|w_{n}-p\right\|+\alpha_{n}\|\gamma f(p)-A p\|+\varphi(1)\left(1-\alpha_{n} \bar{\gamma}\right)\left\|w_{n}-p\right\| \\
& =\left(1-\alpha_{n}(\bar{\gamma} \varphi(1)-\gamma \alpha)\right)\left\|w_{n}-p\right\|+\alpha_{n}(\bar{\gamma} \varphi(1)-\gamma \alpha) \frac{\|\gamma f(p)-A p\|}{(\bar{\gamma} \varphi(1)-\gamma \alpha)}
\end{aligned}
$$


It follows from induction that

$$
\left\|w_{n+1}-p\right\| \leq \max \left\{\left\|w_{0}-p\right\|, \frac{\|\gamma f(p)-A(p)\|}{\bar{\gamma} \varphi(1)-\gamma \alpha}\right\}, \quad n \geq 0 .
$$

Thus, both $\left\{u_{n}\right\}$ and $\left\{w_{n}\right\}$ are bounded. We observe that

$$
u_{n+1}=\alpha_{n+1} f\left(w_{n+1}\right)+\left(I-\alpha_{n+1} A\right) w_{n+1}=\sigma_{n} f\left(T\left(t_{n}\right) u_{n}\right)+\left(I-\sigma_{n} A\right) T\left(t_{n}\right) u_{n} .
$$

Thus, Corollary 3.4 implies that $\left\{u_{n}\right\}$ converges strongly to some point $\tilde{x}$. In this case, we also have

$$
\left\|w_{n}-\tilde{x}\right\| \leq\left\|w_{n}-u_{n}\right\|+\left\|u_{n}-\tilde{x}\right\|=\alpha_{n}\left\|\gamma f\left(w_{n}\right)-A w_{n}\right\|+\left\|u_{n}-\tilde{x}\right\| \rightarrow 0 .
$$

Hence, the sequence $\left\{w_{n}\right\}$ converges strongly to some point $\tilde{x}$. This completes the proof. $\square$

By Lemma 2.2, we obtain the following corollary.

Corollary 3.6. Let E be a uniformly convex Banach space which admits a weakly continuous duality mapping $J_{\phi}$ with gauge $\phi$ such that $\phi$ is invariant on $[0,1]$. Let $C$ be a nonempty closed convex subset of $E$ and $\mathcal{S}=\{T(s): s \geq 0\}$ a nonexpansive semigroup from $C$ into itself such that $F(\mathcal{S}) \neq \emptyset$.

Let $f \in \Pi_{E}$, and let $A$ be a strongly positive linear bounded operator with a coefficient $0<\gamma<\frac{\bar{\gamma} \varphi(1)}{\alpha}$ and $0<\gamma<\frac{\bar{\gamma} \varphi(1)}{\alpha}$. Let the sequence $\left\{x_{n}\right\}$ be generated by the following:

$$
\left\{\begin{array}{l}
x_{0}=x \in E \\
y_{n}=\beta_{n} x_{n}+\left(1-\beta_{n}\right) \frac{1}{t_{n}} \int_{0}^{t_{n}} T(s) x_{n} \mathrm{~d} s \\
x_{n+1}=\alpha_{n} \gamma f\left(x_{n}\right)+\left(I-\alpha_{n} A\right) y_{n}, \quad n \geq 0
\end{array}\right.
$$

where $\left\{\alpha_{n}\right\} \subset(0,1)$ and $\left\{\beta_{n}\right\} \subset[0,1]$ are real sequences satisfying the following conditions:

(C1) $\lim _{n \rightarrow \infty} \alpha_{n}=0$ and $\sum_{n=1}^{\infty} \alpha_{n}=\infty$

(C2) $\lim _{n \rightarrow \infty} \beta_{n}=0$,

(C3) $\lim _{n \rightarrow \infty} t_{n}=\infty$.

Then $\left\{x_{n}\right\}$ converges strongly to $\tilde{x}$ that is obtained in Lemma 3.2.

Setting $E \equiv H$ and $\beta_{n} \equiv 0$ a real Hilbert space in Corollary 3.6, we have the following result.

Corollary 3.7. [[15], Theorem 3.2] Let $H$ be a real Hilbert space. Let $C$ be a nonempty closed convex subset of $E$ and $\mathcal{S}=\{T(s): s \geq 0\}$ a nonexpansive semigroup from $C$ into itself such that $F(\mathcal{S}) \neq \emptyset$. Let $f \in \Pi_{E}$, and let $A$ be a strongly positive linear bounded operator with a coefficient $\bar{\gamma}>0$ and $0<\gamma<\frac{\bar{\gamma}}{\alpha}$. Let the sequence $\left\{x_{n}\right\}$ be generated by the following:

$$
\left\{\begin{array}{l}
x_{0}=x \in E \\
x_{n+1}=\alpha_{n} \gamma f\left(x_{n}\right)+\left(I-\alpha_{n} A\right) \frac{1}{t_{n}} \int_{0}^{t_{n}} T(s) x_{n} \mathrm{~d} s, \quad n \geq 0
\end{array}\right.
$$


where $\left\{\alpha_{n}\right\} \subset(0,1)$ is a real sequences satisfying the following conditions:

(C1) $\lim _{n \rightarrow \infty} \alpha_{n}=0$ and $\sum_{n=1}^{\infty} \alpha_{n}=\infty$

(C2) $\lim _{n \rightarrow \infty} t_{n}=\infty$.

Then $\left\{x_{n}\right\}$ converges strongly to $\tilde{x}$ that is obtained in Lemma 3.2. Then $\left\{x_{n}\right\}$ converges strongly to $\tilde{x}$ which solves the variational inequality (1.12).

\section{Acknowledgements}

The project was supported by Naresuan university, Thailand.

\section{Authors' contributions}

All authors contributed equally and significantly in writing this paper. All authors read and approved the final manuscript.

\section{Competing interests}

The authors declare that they have no competing interests.

Received: 6 July 2011 Accepted: 7 November 2011 Published: 7 November 2011

\section{References}

1. Browder, FE: Fixed point theorems for noncompact mappings in Hilbert spaces. Proc Natl Acad Sci USA. 53, 1272-1276 (1965). doi:10.1073/pnas.53.6.1272

2. Reich, S: Strong convergence theorems for resolvents of accretive operators in Banach spaces. J Math Anal Appl. 75 287-292 (1980). doi:10.1016/0022-247X(80)90323-6

3. $\mathrm{Xu}, \mathrm{HK}$ : Strong convergence of an iterative method for nonexpansive and accretive operators. J Math Anal Appl. 314, 631-643 (2006). doi:10.1016/j.jmaa.2005.04.082

4. Deutsch, F, Yamada, I: Minimizing certain convex functions over the intersection of the fixed point sets of nonexpansive mappings. Numer Funct Anal Optim. 19,33-56 (1998)

5. Xu, HK: An iterative approach to quadratic optimization. J Optim Theory Appl. 116, 659-678 (2003). doi:10.1023/ A:1023073621589

6. Xu, HK: Iterative algorithms for nonlinear operators. J Lond Math Soc. 66, 240-256 (2002). doi:10.1112/ S0024610702003332

7. Browder, FE: Convergence theorems for sequences of nonlinear operators in Banach spaces. Mathematische Zeitschrift. 100, 201-225 (1967). doi:10.1007/BF01109805

8. Wangkeeree, R, Petrot, N, Wangkeeree, R: The general iterative methods for nonexpansive mappings in Banach spaces. J Glob Optim. 51, 27-46 (2011). doi:10.1007/s10898-010-9617-6

9. Moudafi, A: Viscosity approximation methods for fixed-points problems. J Math Anal Appl. 241, 46-55 (2000) doi:10.1006/jmaa.1999.6615

10. Plubtieng, S, Thammathiwat, T: A viscosity approximation method for equilibrium problems, fixed point problems of nonexpansive mappings and a general system of variational inequalities. J Glob Optim. 46(3):447-464 (2010). doi:10.1007/s10898-009-9448-5

11. Xu, HK: Viscosity approximation methods for nonexpansive mappings. J Math Anal Appl. 298, 279-291 (2004). doi:10.1016/j.jmaa.2004.04.059

12. Marino, G, Xu, HK: A general iterative method for nonexpansive mapping in Hilbert spaces. J Math Anal Appl. 318 43-52 (2006). doi:10.1016/j.jmaa.2005.05.028

13. Aoyama, K, Kimura, Y, Takahashi, W, Toyoda, M: Approximation of common fixed points of a countable family of nonexpansive mappings in a Banach space. Nonlinear Anal. 67, 2350-2360 (2007). doi:10.1016/j.na.2006.08.032

14. Jung, JS: Iterative approaches to common fixed points of nonexpansive mappings in Banach spaces. J Math Anal Appl. 302, 509-520 (2005). doi:10.1016/j.jmaa.2004.08.022

15. Li, S, Li, L, Su, Y: General iterative methods for a one-parameter nonexpansive semigroup in Hilbert space. Nonlinear Anal. 70, 3065-3071 (2009). doi:10.1016/..na.2008.04.007

16. Takahashi, W: Nonlinear Functional Analysis. Yokohama Publishers, Yokohama (2000)

17. Lim, TC, Xu, HK: Fixed point theorems for asymptotically nonexpansive mappings. Nonlinear Anal. 22, 1345-1355 (1994). doi:10.1016/0362-546X(94)90116-3

18. Aleyner, $\mathrm{A}$, Censor, $\mathrm{Y}$ : Best approximation to common fixed points of a semigroup of nonexpansive operators. Journal of Nonlinear and Convex Analysis. 6, 137-151 (2005)

19. Chen, $R$, Song, Y: Convergence to common fixed point of nonexpansive semigroups. J Comput Appl Math. 200 566-575 (2007). doi:10.1016/j.cam.2006.01.009

doi:10.1186/1687-1812-2011-76

Cite this article as: Wangkeeree and Preechasilp: The modified general iterative methods for nonexpansive semigroups in banach spaces ${ }^{\dagger}$. Fixed Point Theory and Applications 2011 2011:76. 